\title{
Prediction of Glasgow Outcome Scale after Barbiturate Coma Therapy in patients suffering from Refractory Intracranial Hypertension after Brain Tumour Surgery: Incorporating Ensemble approach into automated Machine Learning
}

\author{
Ali Haider Bangash ${ }^{1,2^{*}}$, Dr Tauseef Ullah $M B B S^{3}$, Professor Dr Inayat Ullah Khan $M B B S, F R C S^{4,5}$, Dr Haris Khan \\ $M B B S^{3}$, Saiqa Zehra ${ }^{1}$
}

${ }^{1}$ STMU Shifa College of Medicine, Islamabad, Pakistan

${ }^{2}$ Member, Working group 3, COST Action EVidence-Based RESearch (EVBRES)

${ }^{3}$ Department of Neurosurgery, Shifa International Hospital, Islamabad, Pakistan

${ }^{4}$ Professor of Neurosurgery, STMU Shifa College of Medicine, Islamabad, Pakistan

${ }^{5}$ Chief of Neurosurgery, Shifa International Hospital, Islamabad, Pakistan

$\S$ All of the listed authors contributed equally.

*Corresponding author: Ali Haider Bangash; Member, Working group 3, COST Action EVidence-Based RESearch (EVBRES); Email: hhaider4@gmail.com

- Refractory intracranial hypertension: $\mathrm{RICH}$

Abbreviations

- Barbiturate coma therapy: BCT

- Machine learning: ML

- Automated ML: aML

- In-hospital mortality: IHM

- Glasgow outcome scale: GOS

- Brain tumor surgery: BTS

- State-of-the-art: SOTA

- Area under the Response-operating Curve: AUROC

- macro-weighted average AUROC: mwA-AUROC

- Linear logistic regression: Llr

- Neural Network: NN

- Random Forest: RF

- Decision Tree: DT

- Development and training time: devT

- At 1 month: $1 \mathrm{M}$

- At 3 months: $3 \mathrm{M}$

- Seconds: $\mathrm{s}$

- eXtreme-Gradient Boosting algorithm: XgBoost

\begin{abstract}
Background: For patients experiencing RICH after being surgically intervened for a brain tumour, BCT provides an efficacious, alternative treatment option. With ML exhibiting undeniable potential in predicting post-interventional outcomes for several ailments, its sub-specialization (aML) was explored to develop models that predict IHM and GOS after BCT in patients exhibiting post-BTS RICH.
\end{abstract}


Methods: Upon the comprehensive dataset of 73 patients exhibiting post-BTS RICH subsequently intervened by BCT as shared by Ryu JA et al. ${ }^{1}$, the current SOTA for $\mathrm{aML}^{2}$ was adopted to develop predictive models. Ensemble approach, where two or more ML algorithmic models are collated to obtain higher discriminative ability in predicting the outcome of interest, was incorporated. mwA-AUROC gauged the discriminating ability of models with the highest value of 1 indicating perfect discriminative classification ability.

Results: Ensemble of Llr, NN and RF predicted GOS at discharge with a perfect mwA-AUROC and devT of $151 \mathrm{~s}$. Llr achieved a perfect mwA-AUROC while predicting GOS 6M with a considerably short devT of only $18 \mathrm{~s}$. GOS $12 \mathrm{M}$ was best predicted with mwA-AUROC of 0.99 by an ensemble of Llr and RF with a devT of $152 \mathrm{~s}$. In a minuscule devT of only $18 \mathrm{~s}$, the XgBoost algorithmic model achieved the perfect mwA-AUROC while predicting IHM.

Conclusion: Our research endeavour, which constitutes the very first attempt to predict outcomes of BCT in patients exhibiting post-BTS RICH by adopting aML, not only achieved perfect, instantaneous results that shall allow optimization of risk stratification protocols in such patients but will also go a long way to underpin the yet-untapped superior statistical authority of aML.

Keywords: Barbiturate coma therapy, Intracranial hypertension, Glasgow outcome scale, Neurosurgical oncology, Automated machine learning, Artificial intelligence

\footnotetext{
${ }^{1}$ Ryu JA, Jung W, Jung YJ, Kwon DY, Kang K, Choi H, Kong DS, Seol HJ, Lee JI. Early prediction of neurological outcome after barbiturate coma therapy in patients undergoing brain tumor surgery. PLoS One. $2019 \mathrm{Apr}$ 17;14(4):e0215280. doi: 10.1371/journal.pone.0215280. PMID: 30995269; PMCID: PMC6469802.

${ }^{2}$ AutoML Compare [Internet]. MLAR Automated Machine Learning. 2021 [cited 19 June 2021]. Available from: https://mljar.com/automl-compare/
} 